\title{
CERTAIN ESTIMATES OF NORMALIZED ANALYTIC FUNCTIONS
}

\author{
SWATI ANAND, NAVEEN KUMAR JAIN, AND SUSHIL KUMAR
}

\begin{abstract}
Let $\phi$ be a normalized convex function defined on open unit disk $\mathbb{D}$. For a unified class of normalized analytic functions which satisfy the second order differential subordination $f^{\prime}(z)+\alpha z f^{\prime \prime}(z) \prec \phi(z)$ for all $z \in \mathbb{D}$, we investigate the distortion theorem and growth theorem. Further, the bounds on initial logarithmic coefficients, inverse coefficient and the second Hankel determinant involving the inverse coefficients are examined.
\end{abstract}

\section{INTRODUCTION}

The class of all normalized analytic functions

$$
f(z)=z+a_{2} z^{2}+a_{3} z^{3}+\cdots
$$

in the unit disk $\mathbb{D}:=\{z \in \mathbb{C}:|z|<1\}$ is denoted by $\mathcal{A}$. Denote by $\mathcal{S}$, the subclass of $\mathcal{A}$ consisting of univalent functions in $\mathbb{D}$. Let $\mathcal{P}$ be the class of analytic functions $p$ defined on $\mathbb{D}$, normalized by the condition $p(0)=1$ and satisfying $\operatorname{Re}(p(z))>0$. Let $f$ and $g$ be analytic in $\mathbb{D}$. Then $f$ is subordinate to $g$, denoted by $f \prec g$, if there exists an analytic function $w$ with $w(0)=0$ and $|w(z)|<1$ for $z \in \mathbb{D}$ such that $f(z)=g(w(z))$. In particular, if $g$ is univalent in $\mathbb{D}$ then $f$ is subordinate to $g$ when $f(0)=g(0)$ and $f(\mathbb{D}) \subseteq g(\mathbb{D})$. In this paper we shall assume that $\phi$ is an analytic function with positive real part in $\mathbb{D}$ and normalized by the conditions $\phi(0)=1$ and $\phi^{\prime}(0)>0$. It is noted that $\phi(\mathbb{D})$ is convex. The function $\phi$ is symmetric with respect to the real axis and it maps $\mathbb{D}$ onto a region starlike with respect to $\phi(0)=1$. The Taylor series representation of the function $\phi$ is given by

$$
\phi(z)=1+B_{1} z+B_{2} z^{2}+B_{3} z^{3}+\cdots,
$$

where $B_{1}>0$. For such $\phi$, Ma and Minda [21] studied the unified subclasses $S^{*}(\phi)$ and $C(\phi)$ of starlike and convex functions respectively, analytically defined as

$$
S^{*}(\phi)=\left\{f \in \mathcal{A}: \frac{z f^{\prime}(z)}{f(z)} \prec \phi(z)\right\} \text { and } \quad C(\phi)=\left\{f \in \mathcal{A}: 1+\frac{z f^{\prime \prime}(z)}{f^{\prime}(z)} \prec \phi(z)\right\} .
$$

The authors investigated the growth, distortion and coefficient estimates for these classes. In particular, the class $S^{*}(\phi)$ reduces to some well known subclasses of starlike fuctions. For example, when $-1 \leq B<A \leq 1, S^{*}[A, B]$ is the class of Janowski starlike functions introduced by Janowski [11]. For $0 \leq \alpha<1, S^{*}[1-2 \alpha,-1]=S_{\alpha}^{*}$ is the class of starlike functions of order $\alpha$, introduced by Robertson [30]. The class $\mathcal{S} \mathcal{L}=\mathcal{S}^{*}(\sqrt{1+z})$, introduced by Sokół and Stankiewicz [35], consists of functions $f \in \mathcal{A}$ such that $z f^{\prime}(z) / f(z)$ lies in the region $\Omega_{L}:=\left\{w \in \mathbb{C}:\left|w^{2}-1\right|<1\right\}$, that is, the right-half of the lemniscate of Bernoulli. Later, Mendiratta et al. [22] introduced the class $\mathcal{S}^{*}{ }_{e}:=\mathcal{S}^{*}\left(e^{z}\right)$ consists of functions $f \in \mathcal{A}$ satisfying the condition $\left|\log \left(z f^{\prime}(z) / f^{\prime}(z)\right)\right|<1$. In 2011, Ali et al. [3](see also [10]) studied

2010 Mathematics Subject Classification. 30C45; 30C50; 30C80.

Key words and phrases. Differential subordination; Growth Theorem; Distortion Theorem; Logarithmic Coefficient; Inverse Coefficient; Hankel determinant. 
the class of all those functions $f \in \mathcal{A}$ which satisfy the following third order differential equation

$$
f(z)+\alpha f^{\prime}(z)+\gamma z^{2} f^{\prime \prime}(z)=g(z)
$$

where the function $g$ is subordinate to a convex function $h$. In [3], the best dominant on all solutions of the differential equation in terms of double integral were obtained. Some certain variations of the class $\mathcal{R}(\alpha, h)=\left\{f \in \mathcal{A}: f^{\prime}(z)+\alpha z f^{\prime \prime}(z) \prec h(z), z \in \mathbb{D}\right\}$, where $h$ is a convex function have been investigated by several authors $[6,23,34,36,38]$. On the basis of the above discussed works, we consider a unified class of all functions $f \in \mathcal{A}$ such that

$$
f^{\prime}(z)+\alpha z f^{\prime \prime}(z) \prec \phi(z)
$$

for $z \in \mathbb{D}$ and where $\alpha \in \mathbb{C}$ with $\operatorname{Re} \alpha \geq 0$. The class of such functions is denoted by $\mathcal{R}(\alpha, \phi)$. Since $f \in R(\alpha, \phi), f^{\prime}(z)+\alpha z f^{\prime \prime}(z) \neq \phi\left(e^{i \theta}\right), \theta \in[0,2 \pi)$, it is observed that

$$
f^{\prime}(z)+\alpha z f^{\prime \prime}(z)=\left[(1-\alpha) f(z)+\alpha\left(z f^{\prime}(z)\right)\right]^{\prime} .
$$

Also, we have $z f^{\prime}(z)=f(z) * \frac{z}{(1-z)^{2}}$ and $f(z)=f(z) * \frac{z}{1-z}$. Thus,

$$
\begin{aligned}
f^{\prime}(z)+\alpha z f^{\prime \prime}(z) & =\left((1-\alpha) f(z) * \frac{z}{1-z}+\alpha f(z) * \frac{z}{(1-z)^{2}}\right)^{\prime} \\
& =\left(f(z) *\left((1-\alpha) \frac{z}{1-z}+\alpha \frac{z}{(1-z)^{2}}\right)\right)^{\prime} .
\end{aligned}
$$

Therefore, we conclude that

$$
\left(f(z) * \frac{z-z^{2}(1-\alpha)}{(1-z)^{2}}\right)^{\prime}-\phi\left(e^{i \theta}\right) \neq 0
$$

or equivalently

$$
\frac{1}{z}\left(f(z) * \frac{z+z^{2}(2 \alpha-1)}{(1-z)^{3}}\right) \neq \phi\left(e^{i \theta}\right)
$$

which is the necessary and sufficient conditions for a function $f \in \mathcal{A}$ to be in the class $\mathcal{R}(\alpha, \phi)$.

In this paper, we compute the distortion, growth inequalities for a function $f$ in the class $\mathcal{R}(\alpha, \phi)$. The sharp bounds on initial logarithmic coefficients for such functions are also obtained. Next, we obtain the bounds on initial inverse coefficients of the function $f \in \mathcal{R}(\alpha, \phi)$ as well as bounds on Fekete Szegö functional and second Hankel determinant.

\section{Distortion and Growth Theorem}

The first theorem proves the distortion theorem of the functions $f$ belonging to $\mathcal{R}(\alpha, \phi)$.

Theorem 2.1. Let $\alpha \in \mathbb{C}$, Re $\alpha \geq 0$ and the function $\phi$ be defined by (1.2). If the function $f \in \mathcal{R}(\alpha, \phi)$, then

$$
1+\sum_{n=1}^{\infty} \frac{\left|B_{n}\right|(-r)^{n}}{n \operatorname{Re} \alpha+1} \leq\left|f^{\prime}(z)\right| \leq 1+\sum_{n=1}^{\infty} \frac{\left|B_{n}\right| r^{n}}{n \operatorname{Re} \alpha+1}
$$

for $|z|<r<1$. The result is sharp. 
We make use of the following lemma in order to prove some of our results.

Lemma 2.2. [7, Lemma 2, p. 192] Let $h$ be a convex function with Re $\gamma \geq 0$. If $p(z)$ is regular in $\mathbb{D}$ and $p(0)=h(0)$, then

$$
p(z)+\frac{z p^{\prime}(z)}{\gamma} \prec h(z)
$$

implies that $p(z) \prec q(z) \prec h(z)$, where

$$
q(z)=\gamma z^{-\gamma} \int_{0}^{z} h(t) t^{\gamma-1} d t
$$

The function $q$ is convex and the best dominant.

Proof of Theorem 2.1. Let the fuction $f$ be in the class $\mathcal{R}(\alpha, \phi)$. Then $f^{\prime}(z)+\alpha z f^{\prime \prime}(z) \prec$ $\phi(z)$. For $p(z)=f^{\prime}(z)$ and $\gamma=1 / \alpha$, Lemma 2.2 yields

$$
f^{\prime}(z) \prec \frac{1}{\alpha} z^{-1 / \alpha} \int_{0}^{z} \phi(t) t^{1 / \alpha-1} d t .
$$

On taking $t=z \zeta^{\alpha}$ in (2), we get

$$
f^{\prime}(z) \prec \int_{0}^{1} \phi\left(z \zeta^{\alpha}\right) d \zeta .
$$

Since the function $\phi$ is symmetric with respect to real axis, $\phi(z)$ has real coefficients. Also $\phi^{\prime}(0)>0$ gives $\phi^{\prime}(x)$ is increasing on $(0,1)$. Thus,

$$
\min _{|z=r|} \operatorname{Re} \phi(z)=\phi(-r) \text { and } \max _{|z=r|} \operatorname{Re} \phi(z)=\phi(r) .
$$

Using (2.3) and (2.2) for $|z|=r$, we have

$$
\begin{aligned}
\left|f^{\prime}(z)\right| \geq \operatorname{Re} f^{\prime}(z) \geq \min _{|z|=r} \operatorname{Re} f^{\prime}(z) \geq \min _{|z|=r} \operatorname{Re} \int_{0}^{1} \phi\left(z \zeta^{\alpha}\right) d \zeta & =\int_{0}^{1} \min _{|z|=r} \operatorname{Re} \phi\left(z \zeta^{\alpha}\right) d \zeta \\
& =\int_{0}^{1} \phi\left(-r \zeta^{\operatorname{Re} \alpha}\right) d \zeta
\end{aligned}
$$

Similarly, we have

$$
\left|f^{\prime}(z)\right| \leq \int_{0}^{1} \phi\left(r \zeta^{\operatorname{Re} \alpha}\right) d \zeta .
$$

Since $\phi\left(z \zeta^{\operatorname{Re} \alpha}\right)=1+B_{1} z \zeta^{\operatorname{Re} \alpha}+B_{2} z^{2} \zeta^{2 \operatorname{Re} \alpha}+\cdots$, a simple calculation yields

$$
\begin{aligned}
\int_{0}^{1} \phi\left(z \zeta^{\operatorname{Re} \alpha}\right) d \zeta & =\int_{0}^{1}\left(1+B_{1} z \zeta^{\operatorname{Re} \alpha}+B_{2} z^{2} \zeta^{2 \operatorname{Re} \alpha}+B_{3} z^{3} \zeta^{3 \operatorname{Re} \alpha}+\cdots\right) d \zeta \\
& =1+\frac{B_{1} z}{\operatorname{Re} \alpha+1}+\frac{B_{2} z^{2}}{2 \operatorname{Re} \alpha+1}+\frac{B_{3} z^{3}}{3 \operatorname{Re} \alpha+1}+\cdots \\
& =1+\sum_{n=1}^{\infty} \frac{B_{n} z^{n}}{n \operatorname{Re} \alpha+1}
\end{aligned}
$$


From (2.4), (2.5) and (2.6) the result follows. The result is sharp for the function $f: \mathbb{D} \rightarrow \mathbb{C}$ defined by

$$
f(z)=z+\sum_{n=1}^{\infty} \frac{B_{n} z^{n+1}}{(n+1)(1+n \operatorname{Re} \alpha)} .
$$

Theorem 2.3. Let $\alpha \in \mathbb{C}$ such that $\operatorname{Re} \alpha \geq 0$ and the function $\phi$ be as in (1.2). Then for the function $f \in R(\alpha, \phi)$, we have

$$
1+\sum_{n=1}^{\infty} \frac{\left|B_{n}\right|(-r)^{n}}{(n+1)(n \operatorname{Re} \alpha+1)} \leq \frac{|f(z)|}{|z|} \leq 1+\sum_{n=1}^{\infty} \frac{\left|B_{n}\right| r^{n}}{(n+1)(n \operatorname{Re} \alpha+1)}(|z|<r<1) .
$$

Proof. Let

$$
H(z)=\int_{0}^{1} \phi\left(z \zeta^{\alpha}\right) d \zeta
$$

and

$$
\Phi_{\alpha}(z)=\int_{0}^{1} \frac{1}{1-z t^{\alpha}} d t=\sum_{n=0}^{\infty} \frac{z^{n}}{1+n \alpha} .
$$

From [32, Theorem 5, p.113], it is noted that $\Phi_{\alpha}(z)$ is convex with $\operatorname{Re} \alpha \geq 0$. Also,

$$
\Phi_{\alpha}(z) * \phi(z)=\left(\sum_{n=0}^{\infty} \frac{z^{n}}{1+n \alpha}\right) *\left(1+\sum_{n=1}^{\infty} B_{n} z^{n}\right)=1+\sum_{n=1}^{\infty}\left(\frac{B_{n}}{1+n \alpha}\right) z^{n} .
$$

It view of above and (2.6), we have

$$
\Phi_{\alpha}(z) * \phi(z)=\int_{0}^{1} \phi\left(z \zeta^{\alpha}\right) d \zeta=H(z) .
$$

Since convolution of two convex functions is convex, the function $H$ is convex and $H(0)=1$. Putting $\gamma=1$ and $h(z)=H(z)$ in Lemma 2.2, we get

$$
p(z) \prec \frac{1}{z} \int_{0}^{z} H(t) d t
$$

Using (2.8), substituting $t=z \sigma$ and $p(z)=f(z) / z$ in (2.9), we have

$$
\frac{f(z)}{z} \prec \int_{0}^{1} \int_{0}^{1} \phi\left(z \sigma \zeta^{\alpha}\right) d \sigma d \zeta
$$

Let $h(z)=f(z) / z$. Then (2.3) together with (2.10) yields

$$
|h(z)| \geq \min _{|z|=r} \operatorname{Re} h(z) \geq \int_{0}^{1} \int_{0}^{1} \min _{|z|=r} \phi\left(z \sigma \zeta^{\alpha}\right) d \sigma d \zeta=\int_{0}^{1} \int_{0}^{1} \phi\left(-r \sigma \zeta^{\operatorname{Re} \alpha}\right) d \sigma d \zeta .
$$

and

$$
|h(z)| \leq \max _{|z|=r} \operatorname{Re} h(z) \leq \int_{0}^{1} \int_{0}^{1} \max _{|z|=r} \phi\left(z \sigma \zeta^{\alpha}\right) d \sigma d \zeta=\int_{0}^{1} \int_{0}^{1} \phi\left(r \sigma \zeta^{\operatorname{Re} \alpha}\right) d \sigma d \zeta
$$


A simple calculation shows that

$$
\begin{aligned}
\int_{0}^{1} \int_{0}^{1} \phi\left(z \sigma \zeta^{\operatorname{Re} \alpha}\right) d \sigma d \zeta & =\int_{0}^{1}\left(1+\sum_{n=1}^{\infty}\left(\frac{B_{n}}{1+n \operatorname{Re} \alpha}\right)(z \sigma)^{n}\right) d \sigma \\
& =1+\sum_{n=1}^{\infty} \frac{B_{n} z^{n}}{(n+1)(1+n \operatorname{Re} \alpha)}
\end{aligned}
$$

Now, the result follows from (2.11), (2.12) and (2.13). The result is sharp for the function $f$ given by $(2.7)$.

Remark 2.4. Letting $\phi(z)=(1-(1-2 \beta) z) /(1-z)$, where $\beta<1$ and $\alpha=1$, Theorem 2.3 reduces to a result due to Silverman [33, Corollary 2, p.250]. Further, for $\phi(z)=$ $(1-(1-2 \beta) z) /(1-z)$, where $\beta<1$ and $\alpha>0$, Theorem 2.5 yields [6, Corollary 3, p.178].

Theorem 2.5. Let $\alpha \in \mathbb{C}$ such that $\operatorname{Re} \alpha \geq 0$ and the function $\phi$ be as in (1.2) such that $f \in R(\alpha, \phi)$. Then

$$
\left|a_{n}\right| \leq \frac{B_{1}}{|n+n(n-1) \alpha|}, \quad \text { for all } \quad n \geq 2 .
$$

Proof. For $p(z)=1+\sum_{n=1}^{\infty} p_{n} z^{n} \in \mathcal{P}$, set $f^{\prime}(z)+\alpha z f^{\prime \prime}(z)=p(z), z \in \mathbb{D}$. Since $f \in R(\alpha, \phi)$, $p(z) \prec \phi(z)$. A simple calculation gives

$$
f^{\prime}(z)+\alpha z f^{\prime \prime}(z)=1+\sum_{n=2}^{\infty}[n+n(n-1) \alpha] a_{n} z^{n-1}=1+\sum_{n=1}^{\infty} p_{n} z^{n} .
$$

On comparing the coefficients of $z^{n-1}$, we get

$$
(n+n(n-1) \alpha) a_{n}=p_{n-1}, \quad \text { for all } n \geq 2 .
$$

By making use of [31, Theorem X, p.70], we get $\left|p_{n}\right| \leq B_{1}$, for all $n \geq 1$. Hence, we get the desired inequality. The inequality (2.14) is sharp for the function $f_{n}$ given by $f_{n}^{\prime}(z)+\alpha z f_{n}^{\prime \prime}(z)=\phi\left(z^{n-1}\right)$.

Remark 2.6. On taking $\phi(z)=(1-(1-2 \beta) z) /(1-z)$, where $\beta<1$ and $\alpha=1$, Theorem 2.5 yields a result due to Silverman [33, Corollary 1, p.250]. Further, letting $\phi(z)=(1-$ $(1-2 \beta) z) /(1-z)$, where $\beta<1$ and $\alpha>0$ Theorem 2.5 reduces to [6, Corollary 2, p.178].

\section{Bounds on Initial Logarithmic Coefficient}

For a function $f \in \mathcal{S}$, the logarithmic coefficients $\gamma_{n}$ are defined by the following series expansion:

$$
\log \frac{f(z)}{z}=2 \sum_{n=1}^{\infty} \gamma_{n} z^{n}, z \in \mathbb{D} \backslash\{0\}, \log 1:=0 .
$$

On comparing the coefficients of $z$ on both the sides, we get the initial logarithmic coefficients

$$
\begin{aligned}
\gamma_{1} & =\frac{1}{2} a_{2}, \quad \gamma_{2}=\frac{1}{2}\left(a_{3}-\frac{1}{2} a_{2}^{2}\right) \\
\gamma_{3} & =\frac{1}{2}\left(a_{4}-a_{2} a_{3}+\frac{1}{3} a_{2}{ }^{3}\right)
\end{aligned}
$$


In 1979, the authors [5] showed that the logarithmic coefficients $\gamma_{n}$ of every function $f \in \mathcal{S}$ satisfy the inequality $\sum_{n=1}^{\infty}\left|\gamma_{n}\right|^{2} \leq \pi^{2} / 6$, where the equality holds if and only if the function $f$ is rotation of the Koebe function $k(z)=z\left(1-e^{i \theta}\right)^{-2}$ for each $\theta$. The $n^{\text {th }}$ logarithmic coefficient of $k(z)$ is $\gamma_{n}=e^{i n \theta} / n$ for each $\theta$ and $n \geq 1$. In [39], the logarithmic coefficients $\gamma_{n}$ of each close-to-convex function $f \in S$ is bounded by $(A \log n) / n$ where $A$ is an absolute constant. In 2018, the authors [4,27] obtained the bounds on logarithmic coefficients of certain subclasses of the class of close-to-convex functions. Recently, Adegani et. al. [1] investigated the bounds for the initial logarithmic coefficients of the generalized classes $S^{*}(\phi)$ and $C(\phi)$. To find the bounds on initial logarithmic coefficient for class $\mathcal{R}(\alpha, \phi)$, we shall use the following two lemmas.

Lemma 3.1. $[25, \mathrm{p} .172]$ Assume that $w$ is a Schwarz function so that $w(z)=\sum_{n=1}^{\infty} c_{n} z^{n}$. Then

$$
\left|c_{1}\right| \leq 1 \quad \text { and } \quad\left|c_{n}\right| \leq 1-\left|c_{1}\right|^{2}, n=2,3, \cdots
$$

Lemma 3.2. [28, Theorem 1] Let $w(z)=\sum_{n=1}^{\infty} c_{n} z^{n}$ be the Schwarz function. Then for any real numbers $q_{1}$ and $q_{2}$, the following sharp inequality holds:

$$
\left|c_{3}+q_{1} c_{1} c_{2}+q_{2} c_{1}^{3}\right| \leq H\left(q_{1} ; q_{2}\right)
$$

where

$$
H\left(q_{1} ; q_{2}\right)= \begin{cases}1, & \text { if }\left(q_{1}, q_{2}\right) \in D_{1} \cup D_{2} \cup\{(2,1)\}, \\ \left|q_{2}\right|, & \text { if }\left(q_{1}, q_{2}\right) \in \cup_{k=1}^{7} D_{k}, \\ \frac{2}{3}\left(\left|q_{1}\right|+1\right)\left(\frac{1+\left|q_{1}\right|}{3\left(\left|q_{1}\right|+1+q_{2}\right)}\right)^{\frac{1}{2}}, & \text { if }\left(q_{1}, q_{2}\right) \in D_{8} \cup D_{9}, \\ \frac{q_{2}}{3}\left(\frac{q_{1}^{2}-4}{q_{1}^{2}-4 q_{2}}\right)\left(\frac{q_{1}^{2}-4}{3\left(q_{2}-1\right)}\right)^{\frac{1}{2}}, & \text { if }\left(q_{1}, q_{2}\right) \in D_{10} \cup D_{11} \backslash\{(2,1)\}, \\ \frac{2}{3}\left(\left|q_{1}\right|-1\right)\left(\frac{\left|q_{1}\right|-1}{3\left(\left|q_{1}\right|-1-q_{2}\right)}\right)^{\frac{1}{2}}, & \text { if }\left(q_{1}, q_{2}\right) \in D_{12}\end{cases}
$$


and for $k=1,2, \cdots 12$, the sets $D_{k}$ are defined as follows:

$$
\begin{aligned}
D_{1}= & \left\{\left(q_{1}, q_{2}\right):\left|q_{1}\right| \leq \frac{1}{2},\left|q_{2}\right| \leq 1\right\}, \\
D_{2}= & \left\{\left(q_{1}, q_{2}\right): \frac{1}{2} \leq\left|q_{1}\right| \leq 2, \frac{4}{27}\left(\left|q_{1}\right|+1\right)^{3}-\left(\left|q_{1}\right|+1\right) \leq q_{2} \leq 1\right\}, \\
D_{3}= & \left\{\left(q_{1}, q_{2}\right):\left|q_{1}\right| \leq \frac{1}{2}, q_{2} \leq-1\right\}, \\
D_{4}= & \left\{\left(q_{1}, q_{2}\right):\left|q_{1}\right| \geq \frac{1}{2}, q_{2} \leq-\frac{2}{3}\left(\left|q_{1}\right|+1\right)\right\}, \\
D_{5}= & \left\{\left(q_{1}, q_{2}\right):\left|q_{1}\right| \leq 2, q_{2} \geq 1\right\}, \\
D_{6}= & \left\{\left(q_{1}, q_{2}\right): 2 \leq\left|q_{1}\right| \leq 4, q_{2} \geq \frac{1}{12}\left(q_{1}^{2}+8\right)\right\}, \\
D_{7}= & \left\{\left(q_{1}, q_{2}\right):\left|q_{1}\right| \geq 4, q_{2} \geq \frac{2}{3}\left(\left|q_{1}\right|-1\right)\right\}, \\
D_{8}= & \left\{\left(q_{1}, q_{2}\right): \frac{1}{2} \leq\left|q_{1}\right| \leq 2,-\frac{2}{3}\left(\left|q_{1}\right|+1\right) \leq q_{2} \leq \frac{4}{27}\left(\left|q_{1}\right|+1\right)^{3}-\left(\left|q_{1}\right|+1\right)\right\}, \\
& D_{9}=\left\{\left(q_{1}, q_{2}\right):\left|q_{1}\right| \geq 2,-\frac{2}{3}\left(\left|q_{1}\right|+1\right) \leq q_{2} \leq \frac{2\left|q_{1}\right|\left(\left|q_{1}\right|+1\right)}{q_{1}^{2}+2\left|q_{1}\right|+4}\right\}, \\
& D_{10}=\left\{\left(q_{1}, q_{2}\right): 2 \leq\left|q_{1}\right| \leq 4, \frac{2\left|q_{1}\right|\left(\left|q_{1}\right|+1\right)}{q_{1}^{2}+2\left|q_{1}\right|+4} \leq q_{2} \leq \frac{1}{12}\left(q_{1}^{2}+8\right)\right\}, \\
& \left\{\left(q_{1}, q_{2}\right):\left|q_{1}\right| \geq 4, \frac{2\left|q_{1}\right|\left(\left|q_{1}\right|-1\right)}{q_{1}^{2}-2\left|q_{1}\right|+4} \leq q_{2} \leq \frac{2}{3}\left(\left|q_{1}\right|-1\right)\right\} . \\
q_{1}^{2}+2\left|q_{1}\right|+4 & \left.\left\{q_{1} \mid q_{2}\right):\left|q_{1}\right| \geq 4\left|q_{1}\right|+4\right) \\
& \left\{\left(\left|q_{1}\right|+1\right),\right.
\end{aligned}
$$

Theorem 3.3. Let $\alpha \in \mathbb{C}$ such that $\operatorname{Re} \alpha \geq 0$ and the function $\phi$ be as in (1.2). Suppose $f \in \mathcal{R}(\alpha, \phi)$, then the initial logarithmic coefficients of $f$ satisfy the following inequalities:

(i) $\left|\gamma_{1}\right| \leq \frac{B_{1}}{4|1+\alpha|}$

(ii)

$$
\left|\gamma_{2}\right| \leq \begin{cases}\frac{B_{1}}{6|1+2 \alpha|}, & \text { if }\left|8(1+\alpha)^{2} B_{2}-3(1+2 \alpha) B_{1}^{2}\right| \leq 8 B_{1}\left|(1+\alpha)^{2}\right| \\ \frac{\left|8(1+\alpha)^{2} B_{2}-3(1+2 \alpha) B_{1}^{2}\right|}{48\left|(1+\alpha)^{2}\right||1+2 \alpha|}, & \text { if }\left|8(1+\alpha)^{2} B_{2}-3(1+2 \alpha) B_{1}^{2}\right|>8 B_{1}\left|(1+\alpha)^{2}\right|\end{cases}
$$

(iii) If $B_{1}, B_{2}, B_{3}$ and $\alpha$ are real numbers, then

$$
\left|\gamma_{3}\right| \leq \frac{B_{1}}{8(1+3 \alpha)} H\left(q_{1} ; q_{2}\right),
$$

where $H\left(q_{1} ; q_{2}\right)$ is given in Lemma 3.2 such that

$$
q_{1}=\frac{2 B_{2}}{B_{1}}-\frac{2 B_{1}(1+3 \alpha)}{3(1+\alpha)(1+2 \alpha)}
$$


and

$$
q_{2}=\frac{B_{3}}{B_{1}}-\frac{2 B_{2}(1+3 \alpha)}{3(1+\alpha)(1+2 \alpha)}+\frac{B_{1}^{2}(1+3 \alpha)}{6(1+\alpha)^{3}} .
$$

The bounds for $\gamma_{1}$ and $\gamma_{2}$ are sharp.

Proof. Let $f(z)=z+\sum_{n=2}^{\infty} a_{n} z^{n} \in \mathcal{R}(\alpha, \phi)$ where $\phi$ is given by $(1.2)$. Then $f^{\prime}(z)+\alpha z f^{\prime \prime}(z)=$ $\phi(w(z))$ for $z \in \mathbb{D}$, where $w(z)=\sum_{n=1}^{\infty} c_{n} z^{n}$ is the Schwarz function. A simple calculation yields

$$
f^{\prime}(z)+\alpha z f^{\prime \prime}(z)=1+B_{1} c_{1} z+\left(B_{1} c_{2}+B_{2} c_{1}^{2}\right) z^{2}+\left(B_{1} c_{3}+2 c_{1} c_{2} B_{2}+B_{3} c_{1}^{3}\right) z^{3}+\cdots .
$$

On comparing the coefficients of $z$, we obtain

$$
\begin{aligned}
2(1+\alpha) a_{2} & =B_{1} c_{1}, \\
3(1+2 \alpha) a_{3} & =B_{1} c_{2}+B_{2} c_{1}^{2} \text { and } \\
4(1+3 \alpha) a_{4} & =B_{1} c_{3}+2 B_{2} c_{1} c_{2}+B_{3} c_{1}^{3} .
\end{aligned}
$$

On substituting the above values of $a_{i}(i=1,2,3)$ in $(3.2)$, we get

$$
\begin{aligned}
\gamma_{1} & =\frac{B_{1} c_{1}}{4(1+\alpha)}, \\
\gamma_{2} & =\frac{8(1+\alpha)^{2} B_{1} c_{2}+\left(8(1+\alpha)^{2} B_{2}-3(1+2 \alpha) B_{1}^{2}\right) c_{1}^{2}}{48(1+\alpha)^{2}(1+2 \alpha)} \\
\gamma_{3} & =\frac{B_{1}}{8(1+3 \alpha)} c_{3}+\left(\frac{B_{2}}{4(1+3 \alpha)}-\frac{B_{1}^{2}}{12(1+\alpha)(1+2 \alpha)}\right) c_{1} c_{2} \\
& +\left(\frac{B_{3}}{8(1+3 \alpha)}-\frac{B_{1} B_{2}}{12(1+\alpha)(1+2 \alpha)}+\frac{B_{1}^{3}}{48(1+\alpha)^{3}}\right) c_{1}^{3} .
\end{aligned}
$$

By using Lemma 3.1, we get the desired best possible estimate on $\gamma_{1}$. The bound is sharp for $\left|c_{1}\right|=1$.

$$
\begin{aligned}
\left|\gamma_{2}\right| & =\left|\frac{B_{1}}{6(1+2 \alpha)} c_{2}+\frac{8(1+\alpha)^{2} B_{2}-3(1+2 \alpha) B_{1}^{2}}{48(1+\alpha)^{2}(1+2 \alpha)} c_{1}^{2}\right| \\
& \leq \frac{B_{1}}{6|1+2 \alpha|}\left|c_{2}\right|+\frac{\left|8(1+\alpha)^{2} B_{2}-3(1+2 \alpha) B_{1}^{2}\right|}{48\left|(1+\alpha)^{2}\right||1+2 \alpha|}\left|c_{1}^{2}\right| \\
& \leq \frac{B_{1}}{6|1+2 \alpha|}\left(1-\left|c_{1}\right|^{2}\right)+\frac{\left.\mid 8(1+\alpha)^{2} B_{2}-3(1+2 \alpha) B_{1}^{2}\right) \mid}{48\left|(1+\alpha)^{2}\right||1+2 \alpha|}\left|c_{1}^{2}\right| \\
& =\frac{B_{1}}{6|1+2 \alpha|}+\left(\frac{\left|8(1+\alpha)^{2} B_{2}-3(1+2 \alpha) B_{1}^{2}\right|}{48\left|(1+\alpha)^{2}\right||1+2 \alpha|}-\frac{B_{1}}{6|1+2 \alpha|}\right)\left|c_{1}^{2}\right| \\
& \leq\left\{\begin{array}{c}
\frac{B_{1}}{6|1+2 \alpha|}, \\
\frac{\left|8(1+\alpha)^{2} B_{2}-3(1+2 \alpha) B_{1}^{2}\right|}{48\left|(1+\alpha)^{2}\right||1+2 \alpha|}, \quad \text { if } \frac{\left|8(1+\alpha)^{2} B_{2}-3(1+2 \alpha) B_{1}^{2}\right|}{48\left|(1+\alpha)^{2}\right||1+2 \alpha|}>\frac{B_{1}}{6|1+2 \alpha|}
\end{array}\right.
\end{aligned}
$$

These bounds are sharp for $\left|c_{1}\right|=0$ and $\left|c_{1}\right|=1$, respectively. 
The third inequality follows by Lemma 3.1. Using Lemma 3.2 for $\gamma_{3}$, we obtain

$$
\begin{aligned}
\left|\gamma_{3}\right| & =\mid \frac{B_{1}}{8(1+3 \alpha)} c_{3}+\left(\frac{B_{2}}{4(1+3 \alpha)}-\frac{B_{1}^{2}}{12(1+\alpha)(1+2 \alpha)}\right) c_{1} c_{2} \\
& +\left(\frac{B_{3}}{8(1+3 \alpha)}-\frac{B_{1} B_{2}}{12(1+\alpha)(1+2 \alpha)}+\frac{B_{1}^{3}}{48(1+\alpha)^{3}}\right) c_{1}^{3} \mid \\
& =\frac{B_{1}}{8(1+3 \alpha)}\left|c_{3}+c_{1} c_{2} q_{1}+c_{1}^{3} q_{2}\right| \\
& \leq \frac{B_{1}}{8(1+3 \alpha)} H\left(q_{1} ; q_{2}\right),
\end{aligned}
$$

where $q_{1}=2\left(\frac{B_{2}}{B_{1}}-\frac{B_{1}(1+3 \alpha)}{3(1+\alpha)(1+2 \alpha)}\right)$ and $q_{2}=\frac{B_{3}}{B_{1}}-\frac{2 B_{2}(1+3 \alpha)}{3(1+\alpha)(1+2 \alpha)}+\frac{B_{1}^{2}(1+3 \alpha)}{6(1+\alpha)^{3}}$. This completes the proof.

On taking $\phi(z)=e^{z}, \phi(z)=\sqrt{1+z}$ and $\phi(z)=(1+z) /(1-z)$, respectively in Theorem 3.3 the following corollaries follows immediately.

Corollary 3.4. Let $\alpha \in \mathbb{C}$ such that $\operatorname{Re} \alpha \geq 0$ and $\phi(z)=e^{z}$. Suppose $f \in \mathcal{R}(\alpha, \phi)$, then the initial logarithmic coefficients of $f$ satisfy the following inequalities:

(i) $\left|\gamma_{1}\right| \leq \frac{1}{4|1+\alpha|}$

(ii) $\left|\gamma_{2}\right| \leq \frac{1}{6|1+2 \alpha|}$

(iii) $\left|\gamma_{3}\right| \leq \frac{1}{8(1+3 \alpha)} H\left(q_{1} ; q_{2}\right)$,

where $H\left(q_{1} ; q_{2}\right)$ is given in Lemma 3.2 such that

$$
q_{1}=\frac{1+3 \alpha(1+2 \alpha)}{3(1+\alpha)(1+2 \alpha)}
$$

and

$$
q_{2}=\frac{1}{6}-\frac{(1+3 \alpha)}{3(1+\alpha)(1+2 \alpha)}+\frac{(1+3 \alpha)}{6(1+\alpha)^{3}} .
$$

Corollary 3.5. Suppose that $f \in \mathcal{R}(\alpha, \phi)$ where $\operatorname{Re} \alpha \geq 0$ and $\phi(z)=\sqrt{1+z}$, then the initial logarithmic coefficients of $f$ satisfy the following inequalities:

(i) $\left|\gamma_{1}\right| \leq \frac{1}{8|1+\alpha|}$

(ii) $\left|\gamma_{2}\right| \leq \frac{1}{12|1+2 \alpha|}$

(iii) $\left|\gamma_{3}\right| \leq \frac{1}{16(1+3 \alpha)} H\left(q_{1} ; q_{2}\right)$,

where $H\left(q_{1} ; q_{2}\right)$ is given in Lemma 3.2 such that

$$
q_{1}=-\frac{1}{2}-\frac{(1+3 \alpha)}{3(1+\alpha)(1+2 \alpha)}
$$

and

$$
q_{2}=\frac{1}{8}+\frac{1+3 \alpha}{12(1+\alpha)(1+2 \alpha)}+\frac{1+3 \alpha}{24(1+\alpha)^{3}} .
$$


Corollary 3.6. Let the function $f \in \mathcal{R}(\alpha, \phi)$ where $\operatorname{Re} \alpha \geq 0$ and $\phi(z)=(1+z) /(1-z)$, then the initial logarithmic coefficients of $f$ satisfy the following inequalities:

$$
\begin{aligned}
& \text { (i) }\left|\gamma_{1}\right| \leq \frac{1}{2|1+\alpha|} \\
& \text { (ii) }\left|\gamma_{2}\right| \leq \frac{1}{3|1+2 \alpha|} \\
& \text { (iii) }\left|\gamma_{3}\right| \leq \frac{1}{4(1+3 \alpha)} H\left(q_{1} ; q_{2}\right), \\
& \text { where } H\left(q_{1} ; q_{2}\right) \text { is given in Lemma 3.2 such that } \\
& \qquad q_{1}=\frac{2(1+3 \alpha(1+2 \alpha))}{3(1+\alpha)(1+2 \alpha)}
\end{aligned}
$$

and

$$
q_{2}=1-\frac{4(1+3 \alpha)}{3(1+\alpha)(1+2 \alpha)}+\frac{2(1+3 \alpha)}{3(1+\alpha)^{3}} .
$$

\section{Inverse Coefficient Estimates}

From the Koebe one quarter theorem, the image of $\mathbb{D}$ under a function $f \in \mathcal{S}$ contains a disk of radius $1 / 4$. Thus for every univalent function $f$ there exist inverse function $f^{-1}$ such that $f^{-1}(f(z))=z$ for $z \in \mathbb{D}$ and $f\left(f^{-1}(\omega)\right)=\omega$ for $|\omega|<r_{0}(f)$ where $r_{0}(f) \geq 1 / 4$. The function $f^{-1}$ has the Taylor series expansion $f^{-1}(\omega)=\omega+A_{2} \omega^{2}+A_{3} \omega^{3}+\cdots$ in some neighborhood of origin. In 1923, Löwner [20] initiated the problem of estimating the coefficients of inverse function and investigated the coefficient estimates for inverse function $f \in \mathcal{S}$. Later on, this lead to the study of inverse coefficient problem for several subclasses of $\mathcal{S}$ by various authors $[2,17,18,19,28]$. In $[12,15]$, authors obtained the initial inverse coefficients for the well known classes $C$ and $S^{*}(\alpha)(0 \leq \alpha \leq 1)$. Recently, Ravichandran and Verma [29] determined the bounds on inverse coefficient for the Janowski starlike functions.

In this section, we shall investigate the bounds on inverse coefficient. The following lemma is needed to obtain the coefficient bounds for the inverse function.

Lemma 4.1. [17, Lemma 3, p.254] If $p(z)=1+p_{1} z+p_{2} z^{2}+\ldots$ is a function in the class $\mathcal{P}$, then for any complex number $\nu$,

$$
\left|p_{2}-\nu p_{1}^{2}\right| \leq 2 \max \{1,|2 \nu-1|\} .
$$

Theorem 4.2. Let $\alpha \in \mathbb{C}$ such that $\operatorname{Re} \alpha \geq 0$ and the function $\phi$ defined by (1.2). If function $f(z)=z+\sum_{n=2}^{\infty} a_{n} z^{n} \in \mathcal{R}(\alpha, \phi)$ and $f^{-1}(\omega)=\omega+\sum_{n=2}^{\infty} A_{n} \omega^{n}$ for all $\omega$ in some neighbourhood of the origin, then

(i) $\left|A_{2}\right| \leq \frac{B_{1}}{2|1+\alpha|}$,

(ii) $\left|A_{3}\right| \leq \frac{B_{1}}{3|1+2 \alpha|} \max \{1,|\mu|\}$, where $\mu=\frac{3 B_{1}(1+2 \alpha)}{2(1+\alpha)^{2}}-\frac{B_{2}}{B_{1}}$

(iii) If $B_{1}, B_{2}, B_{3}$ and $\alpha$ are real numbers, then

$$
\left|A_{4}\right| \leq \frac{B_{1}}{4(1+3 \alpha)} H\left(q_{1} ; q_{2}\right)
$$


where $H\left(q_{1} ; q_{2}\right)$ is given in Lemma 3.2 such that

$$
q_{1}=2\left(\frac{B_{2}}{B_{1}}-\frac{5 B_{1}(1+3 \alpha)}{3(1+\alpha)(1+2 \alpha)}\right)
$$

and

$$
q_{2}=\frac{B_{3}}{B_{1}}-\frac{10 B_{2}(1+3 \alpha)}{3(1+\alpha)(1+2 \alpha)}+\frac{5 B_{1}^{2}(1+3 \alpha)}{2(1+\alpha)^{3}} .
$$

Proof. Let $f \in \mathcal{R}(\alpha, \phi)$. Then

$$
f^{\prime}(z)+\alpha z f^{\prime \prime}(z)=\phi(w(z))
$$

where $w(z)$ is the analytic function $w$ with $w(0)=0$ and $|w(z)|<1$. Let

$$
p(z)=\frac{1+w(z)}{1-w(z)}=1+p_{1} z+p_{2} z^{2}+p_{3} z^{3}+\cdots .
$$

Since $w: \mathbb{D} \rightarrow \mathbb{D}$ is analytic thus $p$ is a function with positive real part and

$$
w(z)=\frac{p(z)-1}{p(z)+1}=\frac{1}{2} p_{1} z+\frac{1}{2}\left(p_{2}-\frac{p_{1}^{2}}{2}\right) z^{2}+\frac{1}{8}\left(p_{1}^{3}-4 p_{1} p_{2}+4 p_{3}\right) z^{3}+\cdots .
$$

Then

$$
\begin{aligned}
\phi(w(z)) & =1+\frac{B_{1} p_{1}}{2} z+\left(\frac{1}{4} B_{2} p_{1}^{2}+\frac{1}{2} B_{1}\left(p_{2}-\frac{1}{2} p_{1}^{2}\right)\right) z^{2} \\
& +\frac{1}{8}\left(\left(B_{1}-2 B_{2}+B_{3}\right) p_{1}^{3}+4\left(-B_{1}+B_{2}\right) p_{1} p_{2}+4 B_{1} p_{3}\right) z^{3}+\cdots
\end{aligned}
$$

Using expressions (4.3) and (4.1), we obtain

$$
\begin{aligned}
2(1+\alpha) a_{2} & =\frac{B_{1} p_{1}}{2} \\
3(1+2 \alpha) a_{3} & =\frac{1}{4} B_{2} p_{1}^{2}+\frac{1}{2} B_{1}\left(p_{2}-\frac{1}{2} p_{1}^{2}\right) \text { and } \\
4(1+3 \alpha) a_{4} & =\frac{1}{8}\left(\left(B_{1}-2 B_{2}+B_{3}\right) p_{1}^{3}+4\left(-B_{1}+B_{2}\right) p_{1} p_{2}+4 B_{1} p_{3}\right) .
\end{aligned}
$$

Since $f^{-1}(\omega)=\omega+A_{2} \omega^{2}+A_{3} \omega^{3}+A_{4} \omega^{4}+\cdots$ in some neighbourhood of origin, so we have $f\left(f^{-1}(\omega)\right)=\omega$. That is

$$
\begin{aligned}
\omega & =f^{-1}(\omega)+a_{2}\left(f^{-1}(\omega)\right)^{2}+a_{3}\left(f^{-1}(\omega)\right)^{3}+\cdots \\
& =\omega+A_{2} \omega^{2}+A_{3} \omega^{3}+A_{4} \omega^{4}+\cdots+a_{2}\left(\omega+A_{2} \omega^{2}+A_{3} \omega^{3}+A_{4} \omega^{4}+\cdots\right)^{2} \\
& +a_{3}\left(\omega+A_{2} \omega^{2}+A_{3} \omega^{3}+A_{4} \omega^{4}+\cdots\right)^{3} .
\end{aligned}
$$

A simple calculation gives the following realtions:

$$
\begin{aligned}
& A_{2}=-a_{2}, \\
& A_{3}=2 a_{2}^{2}-a_{3} \text { and } \\
& A_{4}=-5 a_{2}^{3}+5 a_{2} a_{3}-a_{4} .
\end{aligned}
$$


On subsituting the values of $a_{i}$ from (4.4) into (4.5) and a simple calculation yields

$$
\begin{aligned}
& A_{2}=\frac{-B_{1}}{4(1+\alpha)} p_{1} \\
& A_{3}=\frac{-B_{1}}{6(1+2 \alpha)} p_{2}+\left(\frac{B_{1}^{2}}{8(1+\alpha)^{2}}-\frac{B_{2}}{12(1+2 \alpha)}+\frac{B_{1}}{12(1+2 \alpha)}\right) p_{1}^{2}
\end{aligned}
$$

In (4.2), on taking $c_{1}=\frac{p_{1}}{2}, c_{2}=\frac{1}{2}\left(p_{2}-\frac{p_{1}^{2}}{2}\right), c_{3}=\frac{1}{8}\left(p_{1}^{3}-4 p_{1} p_{2}+4 p_{3}\right)$ and so on we get,

$$
\begin{aligned}
2(1+\alpha) a_{2} & =B_{1} c_{1}, \\
3(1+2 \alpha) a_{3} & =B_{1} c_{2}+B_{2} c_{1}^{2} \quad \text { and } \\
4(1+3 \alpha) a_{4} & =B_{1} c_{3}+2 B_{2} c_{1} c_{2}+B_{3} c_{1}^{3} .
\end{aligned}
$$

On substituting the values of $a_{i}$ from (4.6) in (4.5), we obtain

$$
\begin{aligned}
A_{4} & =\frac{-B_{1}}{4(1+3 \alpha)} c_{3}+\left(\frac{5 B_{1}^{2}}{6(1+\alpha)(1+2 \alpha)}-\frac{B_{2}}{4(1+3 \alpha)}\right) c_{1} c_{2} \\
& +\left(\frac{-B_{3}}{4(1+3 \alpha)}+\frac{5 B_{1} B_{2}}{6(1+\alpha)(1+2 \alpha)}+\frac{-5 B_{1}^{3}}{8(1+\alpha)^{3}}\right) c_{1}^{3} .
\end{aligned}
$$

Since $\left|p_{1}\right| \leq 2$, we have

Consider

$$
\left|A_{2}\right| \leq \frac{B_{1}}{2|1+\alpha|}
$$

$$
\left|A_{3}\right|=\frac{B_{1}}{6|1+2 \alpha|}\left|p_{2}-\left(\frac{3 B_{1}(1+2 \alpha)}{4(1+\alpha)^{2}}-\frac{B_{2}}{2 B_{1}}+\frac{1}{2}\right) p_{1}^{2}\right| .
$$

Then by Lemma 4.1, we get the desired estimate. Using Lemma 3.2 for $A_{4}$, we obtain

$$
\begin{aligned}
\left|A_{4}\right| & =\mid \frac{-B_{1}}{4(1+3 \alpha)} c_{3}+\left(\frac{-B_{2}}{2(1+3 \alpha)}+\frac{5 B_{1}^{2}}{6(1+\alpha)(1+2 \alpha)}\right) c_{1} c_{2} \\
& +\left(\frac{-B_{3}}{4(1+3 \alpha)}+\frac{5 B_{1} B_{2}}{6(1+\alpha)(1+2 \alpha)}-\frac{5 B_{1}^{3}}{8(1+\alpha)^{3}}\right) c_{1}^{3} \mid \\
& =\frac{B_{1}}{4(1+3 \alpha)}\left|c_{3}+c_{1} c_{2} q_{1}+c_{1}^{3} q_{2}\right| \\
& \leq \frac{B_{1}}{4(1+3 \alpha)} H\left(q_{1} ; q_{2}\right),
\end{aligned}
$$

where $q_{1}=2\left(\frac{B_{2}}{B_{1}}-\frac{5 B_{1}(1+3 \alpha)}{3(1+\alpha)(1+2 \alpha)}\right)$ and $q_{2}=\frac{B_{3}}{B_{1}}-\frac{10 B_{2}(1+3 \alpha)}{3(1+\alpha)(1+2 \alpha)}+\frac{5 B_{1}^{2}(1+3 \alpha)}{2(1+\alpha)^{3}}$.

The following corollaries are an immediate consequence of the Theorem 4.2 for $\phi(z)=e^{z}$, $\phi(z)=\sqrt{1+z}$ and $\phi(z)=(1+z) /(1-z)$, respectively.

Corollary 4.3. Let $\alpha \in \mathbb{C}$ such that $\operatorname{Re} \alpha \geq 0$ and $\phi(z)=e^{z}$. If the function $f(z)=$ $z+\sum_{n=2}^{\infty} a_{n} z^{n} \in \mathcal{R}(\alpha, \phi)$ and $f^{-1}(\omega)=\omega+\sum_{n=2}^{\infty} A_{n} \omega^{n}$ for all $\omega$ in some neighbourhood of the origin, then 
(i) $\left|A_{2}\right| \leq \frac{1}{2|1+\alpha|}$,

(ii) $\left|A_{3}\right| \leq \frac{1}{3|1+2 \alpha|} \max \left\{1,\left|\frac{3(1+2 \alpha)}{2(1+\alpha)^{2}}-\frac{1}{2}\right|\right\}$

(iii) $\left|A_{4}\right| \leq \frac{1}{4(1+3 \alpha)} H\left(q_{1} ; q_{2}\right)$,

where $\alpha$ is real and $H\left(q_{1} ; q_{2}\right)$ is given in Lemma 3.2 such that

$$
q_{1}=\frac{-7+3 \alpha(-7+2 \alpha)}{3(1+\alpha)(1+2 \alpha)}
$$

and

$$
q_{2}=\frac{1}{6}-\frac{5(1+3 \alpha)}{3(1+\alpha)(1+2 \alpha)}+\frac{5(1+3 \alpha)}{2(1+\alpha)^{3}} .
$$

Corollary 4.4. Suppose that the function $f(z)=z+\sum_{n=2}^{\infty} a_{n} z^{n} \in \mathcal{R}(\alpha, \phi)$, where $\operatorname{Re} \alpha \geq 0$ and $\phi(z)=\sqrt{1+z}$ and $f^{-1}(\omega)=\omega+\sum_{n=2}^{\infty} A_{n} \omega^{n}$ for all $\omega$ in some neighbourhood of the origin, then

$$
\begin{aligned}
& \text { (i) }\left|A_{2}\right| \leq \frac{1}{4|1+\alpha|}, \\
& \text { (ii) }\left|A_{3}\right| \leq \frac{1}{6|1+2 \alpha|} \max \left\{1,\left|\frac{3(1+2 \alpha)}{4(1+\alpha)^{2}}+\frac{1}{4}\right|\right\}, \\
& \text { (iii) }\left|A_{4}\right| \leq \frac{1}{8(1+3 \alpha)} H\left(q_{1} ; q_{2}\right),
\end{aligned}
$$
where $\alpha$ is real and $H\left(q_{1} ; q_{2}\right)$ is given in Lemma 3.2 such that

$$
q_{1}=-\frac{1}{2}-\frac{5(1+3 \alpha)}{3(1+\alpha)(1+2 \alpha)}
$$

and

$$
q_{2}=\frac{1}{8}+\frac{5(1+3 \alpha)}{12(1+\alpha)(1+2 \alpha)}+\frac{5(1+3 \alpha)}{8(1+\alpha)^{3}}
$$

\section{Hankel Determinant}

The problem involving coefficient bounds have attracted the interest of many authors in particular to second Hankel determinants and Fekete Szegö functional. The coefficient functional $\left|a_{3}-\mu a_{2}^{2}\right|$ where $\mu$ is a complex number is called the Fekete Szegö functional. The Fekete Szegö problem involves maximizing the functional $\left|a_{3}-\mu a_{2}^{2}\right|$. Some authors have investigated the Fekete Szegö problem for the coefficients of inverse function $[2,24,37]$. The Hankel determinant $\left|H_{2}(1)\right|=\left|a_{3}-a_{2}^{2}\right|$ is a particular case of the Fekete Szegö functional and $H_{2}(2)=\left|a_{2} a_{4}-a_{2}^{3}\right|$ is called the second Hankel determinants. In 2013, Lee et. al. [16] obtained the bounds for the second Hankel determinant for the unified class of MaMinda starlike and convex functions. The authors [37] estimated the bounds on second Hankel determinant for the class of strongly convex functions of order $\alpha$ using the inverse coefficients. One may refer to $[14,8,13,9,26]$ for more details. In the present section, we shall determine the Fekete Szegö functional for the inverse coefficient. 
Theorem 5.1. Suppose $\alpha \in \mathbb{C}$ such that $\operatorname{Re} \alpha \geq 0$ and the function $\phi$ defined by (1.2). Let $f \in \mathcal{R}(\alpha, \phi)$ and $f^{-1}(\omega)=\omega+\sum_{n=2}^{\infty} A_{n} \omega^{n}$ for all $\omega$ in some neighbourhood of the origin. Then for any complex number $\mu$, we have

$$
\left|A_{3}-\mu A_{2}^{2}\right| \leq \frac{B_{1}}{3|1+2 \alpha|} \max \left\{1,\left|\frac{3 B_{1}(1+2 \alpha)}{4(1+\alpha)^{2}}(\mu-2)+\frac{B_{2}}{B_{1}}\right|\right\} .
$$

Proof. In view of euqations (4.4) and (4.5), we get

$$
\begin{aligned}
\left|A_{3}-\mu A_{2}^{2}\right| & =\left|\frac{-B_{1}}{6(1+2 \alpha)} p_{2}+\left(\left(\frac{B_{1}^{2}}{8(1+\alpha)^{2}}+\frac{B_{1}-B_{2}}{12(1+2 \alpha)}\right)-\mu \frac{B_{1}^{2}}{16(1+\alpha)^{2}}\right) p_{1}^{2}\right| \\
& =\left|\frac{B_{1}}{6(1+2 \alpha)}\left(p_{2}+\left(\frac{-3 B_{1}(1+2 \alpha)}{4(1+\alpha)^{2}}+\frac{B_{2}}{2 B_{1}}-\frac{1}{2}+\mu \frac{3 B_{1}(1+2 \alpha)}{8(1+\alpha)^{2}}\right) p_{1}^{2}\right)\right| \\
& =\left|\frac{B_{1}}{6(1+2 \alpha)}\left(p_{2}-\nu p_{1}^{2}\right)\right|
\end{aligned}
$$

where $\nu=\frac{3 B_{1}(1+2 \alpha)}{8(1+\alpha)^{2}}(2-\mu)-\frac{B_{2}}{2 B_{1}}+\frac{1}{2}$. By Lemma 4.1, we get the required result.

Theorem 5.2. Let $\alpha \in \mathbb{C}$ such that $\operatorname{Re} \alpha \geq 0$ and the function $\phi$ defined by (1.2). Suppose function $f$ in $\mathcal{R}(\alpha, \phi)$ and $f^{-1}(\omega)=\omega+\sum_{n=2}^{\infty} A_{n} \omega^{n}$ for all $\omega$ in some neighbourhood of the origin.

(i) If $B_{1}, B_{2}$ and $B_{3}$ satisfy the conditions

$$
4 d_{2} \leq d_{3}, \quad d_{1} \leq \frac{B_{1}|1+\alpha|}{9\left|(1+2 \alpha)^{2}\right|},
$$

then

$$
\left|A_{2} A_{4}-A_{3}^{2}\right| \leq \frac{B_{1}^{2}}{9\left|(1+2 \alpha)^{2}\right|} .
$$

(ii) If $B_{1}, B_{2}$ and $B_{3}$ satisfy the conditions

$$
4 d_{2} \geq d_{3}, \quad d_{1}-\frac{d_{2}}{2}-\frac{B_{1}}{16|1+3 \alpha|} \geq 0
$$

or

$$
4 d_{2} \leq d_{3}, \quad d_{1} \geq \frac{B_{1}|1+\alpha|}{9\left|(1+2 \alpha)^{2}\right|},
$$

then

$$
\left|A_{2} A_{4}-A_{3}^{2}\right| \leq \frac{B_{1} d_{1}}{|1+\alpha|} .
$$

(iii) If $B_{1}, B_{2}$ and $B_{3}$ satisfy the conditions

$$
4 d_{1}>d_{3}, d_{1}-\frac{d_{2}}{2}-\frac{B_{1}}{16|1+3 \alpha|} \leq 0,
$$


then

$$
\left|A_{2} A_{4}-A_{3}^{2}\right| \leq \frac{\frac{B_{1}}{16|1+\alpha|}\left(\frac{16 B_{1}|1+\alpha|}{9\left|(1+2 \alpha)^{2}\right|} d_{1}-\frac{B_{1}}{|1+3 \alpha|} d_{2}-4 d_{2}^{2}-\frac{B_{1}^{2}}{16\left|(1+3 \alpha)^{2}\right|}\right)}{d_{1}-d_{2}-\frac{B_{1}}{8|1+3 \alpha|}+\frac{B_{1}|1+\alpha|}{9\left|(1+2 \alpha)^{2}\right|}},
$$

where

$$
\begin{aligned}
& d_{1}=\frac{B_{1}^{3}}{16\left|(1+\alpha)^{3}\right|}+\frac{B_{2}^{2}|1+\alpha|}{9 B_{1}\left|(1+2 \alpha)^{2}\right|}+\frac{B_{1}\left|B_{2}\right|}{12|1+\alpha||1+2 \alpha|}+\frac{\left|B_{3}\right|}{8|1+3 \alpha|} \\
& d_{2}=\frac{B_{1}^{2}}{12|1+\alpha||1+2 \alpha|}+\frac{\left|B_{2}\right|}{4|1+3 \alpha|}+\frac{2\left|B_{2}\right||1+\alpha|}{9\left|(1+2 \alpha)^{2}\right|}
\end{aligned}
$$

and

$$
d_{3}=\frac{8 B_{1}|1+\alpha|}{9\left|(1+2 \alpha)^{2}\right|}-\frac{B_{1}}{2|1+3 \alpha|} .
$$

In the proof of this result, the following lemma is needed.

Lemma 5.3. [17, Lemma 2, p.254] If $p(z)=1+\sum_{n=1}^{\infty} p_{n} z^{n} \in \mathcal{P}$, then

$$
\begin{aligned}
& 2 p_{2}=p_{1}^{2}+x\left(4-p_{1}^{2}\right) \\
& 4 p_{3}=p_{1}^{3}+2 p_{1}\left(4-p_{1}^{2}\right) x-p_{1}\left(4-p_{1}^{2}\right) x^{2}+2\left(4-p_{1}^{2}\right)\left(1-|x|^{2}\right) z
\end{aligned}
$$

for some $x$ and $z$ such that $|x| \leq 1$ and $|z| \leq 1$.

Remark 5.4. For real numbers $P, Q$ and $R$, a standard computation gives

$$
\max _{0 \leq t \leq 4}\left(P t^{2}+Q t+R\right)= \begin{cases}R, & Q \leq 0, P \leq-Q / 4 \\ 16 P+4 Q+R, & Q \geq 0, P \geq-Q / 8 \text { or } Q \leq 0, P \geq-Q / 4 \\ \frac{4 P R-Q^{2}}{4 P}, & Q>0, P \leq-Q / 8\end{cases}
$$

Proof of Theorem 5.2. It follows from equations (4.4) and (4.5) that

$$
\begin{aligned}
A_{2} A_{4}-A_{3}^{2} & =\frac{-B_{1} p_{1}}{4(1+\alpha)}\left(\frac{-B_{1}}{8(1+3 \alpha)} p_{3}+\left(\frac{5 B_{1}^{2}}{24(1+\alpha)(1+2 \alpha)}+\frac{B_{1}-B_{2}}{8(1+3 \alpha)}\right) p_{1} p_{2}\right. \\
& \left.+\left(\frac{-5 B_{1}^{3}}{6(1+\alpha)^{3}}-\frac{5 B_{1}\left(B_{2}-B_{1}\right)}{48(1+\alpha)(1+2 \alpha)}-\frac{B_{1}-2 B_{2}+B_{3}}{32(1+3 \alpha)}\right) p_{1}^{3}\right) \\
& -\left(\left(\frac{B_{1}^{2}}{8(1+\alpha)^{2}}+\frac{\left(B_{1}-B_{2}\right)}{12(1+2 \alpha)}\right) p_{1}^{2}-\frac{B_{1} p_{2}}{6(1+2 \alpha)}+\right)^{2} \\
& =p_{1}^{4}\left(\frac{B_{1}^{4}}{256(1+\alpha)^{4}}+\frac{B_{1}^{2}\left(B_{1}-B_{2}\right)}{192(1+\alpha)^{2}(1+2 \alpha)}+\frac{B_{1}\left(B_{1}-2 B_{2}+B_{3}\right)}{128(1+\alpha)(1+3 \alpha)}\right. \\
& \left.-\frac{\left(B_{1}-B_{2}\right)^{2}}{144(1+2 \alpha)^{2}}\right)+\frac{B_{1}^{2} p_{1} p_{3}}{32(1+\alpha)(1+3 \alpha)}-\frac{B_{1}^{2} p_{2}^{2}}{36(1+2 \alpha)^{2}} \\
& +p_{1}^{2} p_{2}\left(\frac{-B_{1}^{3}}{96(1+\alpha)^{2}(1+2 \alpha)}-\frac{B_{1}\left(B_{2}-B_{1}\right)}{32(1+\alpha)(1+3 \alpha)}+\frac{B_{1}\left(B_{1}-B_{2}\right)}{36(1+2 \alpha)^{2}}\right) .
\end{aligned}
$$


In view of Lemma 5.3, we obtain

$$
\begin{aligned}
A_{2} A_{4}-A_{3}^{2} & =\frac{B_{1}}{16(1+\alpha)}\left[p_{1}^{4}\left(\frac{B_{1}^{3}}{16(1+\alpha)^{3}}-\frac{B_{2}^{2}(1+\alpha)}{9 B_{1}(1+2 \alpha)^{2}}-\frac{B_{1} B_{2}}{12(1+\alpha)(1+2 \alpha)}+\frac{B_{3}}{8(1+3 \alpha)}\right)\right. \\
& +2 p_{1}^{2}\left(4-p_{1}^{2}\right) x\left(-\frac{B_{1}^{2}}{24(1+\alpha)(1+2 \alpha)}+\frac{B_{2}}{8(1+3 \alpha)}-\frac{B_{2}(1+\alpha)}{9(1+2 \alpha)^{2}}\right) \\
& +\left(4-p_{1}^{2}\right) x^{2} p_{1}^{2}\left(\frac{-B_{1}}{8(1+3 \alpha)}\right)-\left(4-p_{1}^{2}\right)^{2} x^{2}\left(\frac{B_{1}(1+\alpha)}{9(1+2 \alpha)^{2}}\right) \\
& \left.+2 p_{1}\left(4-p_{1}^{2}\right)\left(1-|x|^{2}\right) z \frac{B_{1}}{8(1+3 \alpha)}\right] .
\end{aligned}
$$

Since $\left|p_{1}\right| \leq 2$ and it can be assumed that $p_{1}>0$ and thus we get that $p_{1} \in[0,2]$. Letting $p_{1}=p$ and $|x|=\gamma$ in the above expression, we get

$$
\begin{aligned}
\left|A_{2} A_{4}-A_{3}^{2}\right| & \leq \frac{B_{1}}{16|1+\alpha|}\left[p^{4}\left(\frac{B_{1}^{3}}{16\left|(1+\alpha)^{3}\right|}+\frac{B_{2}^{2}|1+\alpha|}{9 B_{1}\left|(1+2 \alpha)^{2}\right|}+\frac{B_{1}\left|B_{2}\right|}{12|1+\alpha||1+2 \alpha|}+\frac{\left|B_{3}\right|}{8|1+3 \alpha|}\right)\right. \\
& +2 p^{2}\left(4-p^{2}\right) \gamma\left(\frac{B_{1}^{2}}{24|1+\alpha||1+2 \alpha|}+\frac{\left|B_{2}\right|}{8|1+3 \alpha|}+\frac{\left|B_{2}\right||1+\alpha|}{9\left|(1+2 \alpha)^{2}\right|}\right) \\
& +\left(4-p^{2}\right) \gamma^{2} p^{2}\left(\frac{B_{1}}{8|1+3 \alpha|}\right)+\left(4-p^{2}\right)^{2} \gamma^{2}\left(\frac{B_{1}|1+\alpha|}{9\left|(1+2 \alpha)^{2}\right|}\right) \\
& \left.+2 p\left(4-p^{2}\right)\left(1-\gamma^{2}\right)|z| \frac{B_{1}}{8|1+3 \alpha|}\right] . \\
& =: G(p, \gamma) .
\end{aligned}
$$

Let $p$ be fixed. Using the first derivative test in the region $\Omega=\{(p, \gamma): 0 \leq p \leq 2,0 \leq$ $\gamma \leq 1\}$ we get that $G(p, \gamma)$ is an increasing function of $\gamma$ where $\gamma \in[0,1]$. Thus for fixed $p \in[0,2]$, we obtain

$$
\begin{aligned}
& \max _{0 \leq \gamma \leq 1} G(p, \gamma)=G(p, 1)=: F(p), \text { where } \\
& F(p)=\frac{B_{1}}{16|1+\alpha|}\left[p ^ { 4 } \left(\frac{B_{1}^{3}}{16\left|(1+\alpha)^{3}\right|}+\frac{B_{2}^{2}|1+\alpha|}{9 B_{1}\left|(1+2 \alpha)^{2}\right|}+\frac{B_{1}\left|B_{2}\right|}{12|1+\alpha||1+2 \alpha|}+\frac{\left|B_{3}\right|}{8|1+3 \alpha|}\right.\right. \\
&\left.-\frac{B_{1}^{2}}{12|1+\alpha||1+2 \alpha|}-\frac{\left|B_{2}\right|}{4|1+3 \alpha|}-\frac{2\left|B_{2}\right||1+\alpha|}{9\left|(1+2 \alpha)^{2}\right|}-\frac{B_{1}}{8|1+3 \alpha|}+\frac{B_{1}|1+\alpha|}{9\left|(1+2 \alpha)^{2}\right|}\right) \\
&+p^{2}\left(\frac{B_{1}^{2}}{3|1+\alpha||1+2 \alpha|}+\frac{\left|B_{2}\right|}{|1+3 \alpha|}+\frac{8\left|B_{2}\right||1+\alpha|}{9\left|(1+2 \alpha)^{2}\right|}+\frac{B_{1}}{2|1+3 \alpha|}-\frac{8 B_{1}|1+\alpha|}{9\left|(1+2 \alpha)^{2}\right|}\right) \\
&\left.+\frac{16 B_{1}|1+\alpha|}{9\left|(1+2 \alpha)^{2}\right|}\right] .
\end{aligned}
$$


Let

$$
\begin{aligned}
P & =\frac{B_{1}}{16|1+\alpha|}\left[\left(\frac{B_{1}^{3}}{16\left|(1+\alpha)^{3}\right|}+\frac{B_{2}^{2}|1+\alpha|}{9 B_{1}\left|(1+2 \alpha)^{2}\right|}+\frac{B_{1}\left|B_{2}\right|}{12|1+\alpha||1+2 \alpha|}+\frac{\left|B_{3}\right|}{8|1+3 \alpha|}\right)\right. \\
& \left.-\left(\frac{B_{1}^{2}}{12|1+\alpha||1+2 \alpha|}+\frac{\left|B_{2}\right|}{4|1+3 \alpha|}+\frac{2\left|B_{2}\right||1+\alpha|}{9\left|(1+2 \alpha)^{2}\right|}\right)+\left(-\frac{B_{1}}{8|1+3 \alpha|}+\frac{B_{1}|1+\alpha|}{9\left|(1+2 \alpha)^{2}\right|}\right)\right] \\
Q & =\frac{B_{1}}{16|1+\alpha|}\left[4\left(\frac{B_{1}^{2}}{12|1+\alpha||1+2 \alpha|}+\frac{B_{2}}{4|1+3 \alpha|}+\frac{2\left|B_{2}\right||1+\alpha|}{9\left|(1+2 \alpha)^{2}\right|}\right)\right. \\
& \left.-\left(\frac{8 B_{1}|1+\alpha|}{9\left|(1+2 \alpha)^{2}\right|}-\frac{B_{1}}{2|1+3 \alpha|}\right)\right] \\
R & =\frac{B_{1}^{2}}{9\left|(1+2 \alpha)^{2}\right|} \text { and } t=p^{2} .
\end{aligned}
$$

Then $F(t)=P t^{2}+Q t+R$. Using the inequality (5.1), we get the required result.

\section{REFERENCES}

[1] E. A. Adegani, N. E. Cho, and M. Jafari, Logarithmic Coefficients for Univalent Functions Defined by Subordination. Mathematics 2019, 7, 408.

[2] R. M. Ali, Coefficients of the inverse of strongly starlike functions, Bull. Malays. Math. Sci. Soc. (2) 26 (2003), no. 1, 63-71.

[3] R. M. Ali, S. K. Lee, K. G. Subramanian, and A. Swaminathan, A third-order differential equation and starlikeness of a double integral operator, Abstr. Appl. Anal. 2011, Art. ID 901235, 10 pp.

[4] M. F. Ali and A. Vasudevarao, On logarithmic coefficients of some close-to-convex functions, Proc. Amer. Math. Soc. 146 (2018), no. 3, 1131-1142.

[5] P. L. Duren and Y. J. Leung, Logarithmic coefficients of univalent functions, J. Analyse Math. 36 (1979), 36-43 (1980).

[6] C. Y. Gao and S. Q. Zhou, Certain subclass of starlike functions, Appl. Math. Comput. 187 (2007), no. $1,176-182$.

[7] D. J. Hallenbeck and S. Ruscheweyh, Subordination by convex functions, Proc. Amer. Math. Soc. 52 (1975), 191-195.

[8] T. Hayami and S. Owa, Generalized Hankel determinant for certain classes, Int. J. Math. Anal. (Ruse) 4 (2010), no. 49-52, 2573-2585.

[9] W. K. Hayman, On the second Hankel determinant of mean univalent functions, Proc. London Math. Soc. (3) 18 (1968), 77-94.

[10] N. K. Jain and S. Yadav, Bohr Radius for Certain Analytic Functions, In: Deo N., Gupta V., Acu A., Agrawal P. (eds) Mathematical Analysis I: Approximation Theory. ICRAPAM 2018. Springer Proceedings in Mathematics \& Statistics, vol 306. Springer, Singapore, 2020.

[11] W. Janowski, Extremal problems for a family of functions with positive real part and for some related families, Ann. Polon. Math. 23 (1970/1971), 159-177.

[12] G. P. Kapoor and A. K. Mishra, Coefficient estimates for inverses of starlike functions of positive order, J. Math. Anal. Appl. 329 (2007), no. 2, 922-934.

[13] V. Kumar, S. Kumar and V. Ravichandran (2020) Third Hankel Determinant for Certain Classes of Analytic Functions. In: Deo N., Gupta V., Acu A., Agrawal P. (eds) Mathematical Analysis I: Approximation Theory. ICRAPAM 2018. Springer Proceedings in Mathematics and Statistics, vol 306, pp 223-231. Springer, Singapore.

[14] S. Kumar, V. Ravichandran and S. Verma, Initial coefficients of starlike functions with real coefficients, Bull. Iranian Math. Soc. 43 (2017), no. 6, 1837-1854.

[15] J. G. Krzyż, R. J. Libera and E. Złotkiewicz, Coefficients of inverses of regular starlike functions, Ann. Univ. Mariae Curie-Skłodowska Sect. A 33 (1979), 103-110 (1981).

[16] S. K. Lee, V. Ravichandran and S. Supramaniam, Bounds for the second Hankel determinant of certain univalent functions, J. Inequal. Appl. 2013, 2013:281, 17 pp. 
[17] R. J. Libera and E. J. Złotkiewicz, Coefficient bounds for the inverse of a function with derivative in $\mathcal{P}$, Proc. Amer. Math. Soc. 87 (1983), no. 2, 251-257.

[18] R. J. Libera and E. J. Złotkiewicz, The coefficients of the inverse of an odd convex function, Rocky Mountain J. Math. 15 (1985), no. 3, 677-683.

[19] R. J. Libera and E. J. Złotkiewicz, Löwner's inverse coefficients theorem for starlike functions, Amer. Math. Monthly 99 (1992), no. 1, 49-50.

[20] K. Lowner, Untersuchungen uber schlichte konforme Abbildungen des Einheitskreises. I, Math. Ann. 89 (1923), no. 1-2, 103121.

[21] W. C. Ma and D. Minda, A unified treatment of some special classes of univalent functions, in Proceedings of the Conference on Complex Analysis (Tianjin, 1992), 157-169, Conf. Proc. Lecture Notes Anal., I, Int. Press, Cambridge, MA.

[22] R. Mendiratta, S. Nagpal and V. Ravichandran, On a subclass of strongly starlike functions associated with exponential function, Bull. Malays. Math. Sci. Soc. 38 (2015), no. 1, 365-386.

[23] M. A. Nasr and M. K. Aouf, Bounded starlike functions of complex order, Proc. Indian Acad. Sci. Math. Sci. 92 (1983), no. 2, 97-102.

[24] A. Naz, S. Kumar and V. Ravichandran, Coefficients of the inverse functions and radius estimates of certain starlike functions, preprint.

[25] Z. Nehari, Conformal mapping, McGraw-Hill Book Co., Inc., New York, Toronto, London, 1952.

[26] J. W. Noonan and D. K. Thomas, On the second Hankel determinant of areally mean $p$-valent functions, Trans. Amer. Math. Soc. 223 (1976), 337-346.

[27] U. Pranav Kumar and A. Vasudevarao, Logarithmic coefficients for certain subclasses of close-to-convex functions, Monatsh. Math. 187 (2018), no. 3, 543-563.

[28] D. V. Prokhorov and J. Szynal, Inverse coefficients for $(\alpha, \beta)$-convex functions, Ann. Univ. Mariae Curie-Skłodowska Sect. A 35 (1981), 125-143 (1984).

[29] V. Ravichandran and S. Verma, Estimates for coefficients of certain analytic functions, Filomat 31 (2017), no. 11, 3539-3552.

[30] M. S. Robertson, Certain classes of starlike functions, Michigan Math. J. 32 (1985), no. 2, 135-140.

[31] W. Rogosinski, On the coefficients of subordinate functions, Proc. London Math. Soc. (2) 48 (1943), $48-82$.

[32] S. Ruscheweyh, New criteria for univalent functions, Proc. Amer. Math. Soc. 49 (1975), 109-115.

[33] H. Silverman, A class of bounded starlike functions, Internat. J. Math. Math. Sci. 17 (1994), no. 2, 249-252.

[34] R. Singh and S. Singh, Convolution properties of a class of starlike functions, Proc. Amer. Math. Soc. 106 (1989), no. 1, 145-152.

[35] J. Sokół and J. Stankiewicz, Radius of convexity of some subclasses of strongly starlike functions, Zeszyty Nauk. Politech. Rzeszowskiej Mat. No. 19 (1996), 101-105.

[36] H. M. Srivastava, D. Răducanu and P. Zaprawa, A certain subclass of analytic functions defined by means of differential subordination, Filomat 30 (2016), no. 14, 3743-3757.

[37] D. K. Thomas and S. Verma, Invariance of the coefficients of strongly convex functions, Bull. Aust. Math. Soc. 95 (2017), no. 3, 436-445.

[38] D. G. Yang and J. L. Liu, A class of analytic functions with missing coefficients, Abstr. Appl. Anal. 2011, Art. ID 456729, 16 pp.

[39] Z. Ye, The logarithmic coefficients of close-to-convex functions, Bull. Inst. Math. Acad. Sin. (N.S.) 3 (2008), no. 3, 445-452.

Department of Mathematics, University of Delhi, Delhi-110 007, India

E-mail address: swati_anand01@yahoo.com

Department of Mathematics, Aryabhatta College, Delhi-110021,India

E-mail address: naveenjain05@gmail.com

Bharati Vidyapeeth's College of Engineering, Delhi-110063, India

E-mail address: sushilkumar16n@gmail.com 